\title{
Quantized Stabilization of Markov Jump Linear Systems via State Feedback
}

\author{
Nan Xiao, Lihua Xie and Minyue Fu
}

\begin{abstract}
This paper addresses the quantized stabilization problem for single-input Markov jump systems. Modedependent and mode-independent quadratic control Lyapunov functions based on the availability of mode information at controller/quantizer are considered for the quantized feedback. Similar to the linear time-invariant case, it is shown that a mode-dependent (respectively, mode-independent) logarithmic quantizer is optimal (coarsest) in the mean square quadratic stability (respectively, strongly mean square quadratic stability) sense for Markov jump systems. Moreover, the sector bound approach is shown to be nonconservative in investigating the corresponding quantized state feedback problem. Under an appropriate definition of quantization coarseness, we also present a method of optimal quantizer design in terms of linear matrix inequalities. Several examples including applications in networked control systems are given to demonstrate the results.
\end{abstract}

\section{INTRODUCTION}

Starting from Kalman [1], quantization has been known to have an undesirable effect on system performance or even stability, and thereby many works have been done in mitigating the effect. In modern networked systems, quantization is an indispensable step which aims at saving limited bandwidth and energy consumption. Elia and Mitter [2] first pointed out that quantization is "useful, if not essential, instead of undesirable", and also indicated that the coarsest quantizer is logarithmic in the sense of quadratic stability for single-input linear time-invariant (LTI) systems. A relationship between the optimal quantization density and unstable eigenvalues of the plant under consideration has been established. Fu and Xie [3] showed that under quadratic stability, quantized stabilization is equivalent to the robust stabilization of an associated system with sectorbound uncertainty and extended the results to multiple-inputmultiple-output (MIMO) systems and output feedback. Based on the result in [3], quantized stabilization is considered in [4] where a quantization error dependent Lyapunov function is adopted which offers a less conservative design.

The packet-drop behavior of an unreliable communication channel is another important issue in networked control systems (NCSs) as it induces information loss and consequently affects the performance or even stability of the closed-loop

This work was supported by Agency for Science, Technology and Research under Grant SERC 0521010037, NSFC of China under Grant 60828006 and NSFC-Guangdong Joint Foundation U0735003.

N. Xiao and L. Xie are with School of Electrical and Electronic Engineering, Nanyang Technological University, Singapore 639798, Singapore xiao0023entu.edu.sg; elhxieentu.edu.sg

$\mathrm{M}$. Fu is with School of Electrical Engineering and Computer Science, University of Newcastle, Callaghan, NSW 2308, Australia minyue. fu@newcastle.edu.au system. There have been many interesting studies on the packet-loss issue; see, e.g. [5], [6], [7], [8] for networked control, [9], [10], [11] for networked estimation, and [12] for a review of recent results. In [5], the stability robustness of NCSs is addressed, where the packet losses are modeled according to an i.i.d. Bernoulli distribution and the control input becomes zero when the data are lost (so-called zerocontrol strategy). The Markov jump systems (MJSs) theory is applied to the $H_{\infty}$ control of NCSs with binary stochastic packet losses in [6]. In [7], bounded consecutive switching or Markovian packet losses are assumed. [8] considered the mean square stabilization over fading channel in the framework of robust control for deterministic systems with stochastic structured model uncertainties. One of the interesting discoveries in [8] is that the supremum of allowable packet-loss rate (probability of erasure) can be given in terms of the unstable poles of the single-input plant under investigation.

As quantization and packet losses co-exist in a NCS, it is natural and reasonable to take them into consideration simultaneously. The stabilization problem over channel containing both quantization and packet losses was first addressed in [13], where the packet-loss process is driven by a binary i.i.d. process. It was shown in [13] that the upper bound of the coarseness can be given by the packet-loss rate and the unstable eigenvalues of the plant.

Note that the results of [13] for the binary i.i.d. packet dropouts are no longer applicable for binary Markovian losses. In this case, the networked systems can be modeled as a general MJS. Therefore, this paper is to answer the following fundamental questions arising from quantized networked control: a) Is the logarithmic quantizer still optimal (coarsest) for MJSs? b) Is the sector bound approach still nonconservative in dealing with quantized stabilization of MJSs? c) How to design the optimal quantizer? This paper reveals that for MJSs with a given quadratic control Lyapunov function (QCLF), the optimal quantizer can be approached by adopting a logarithmic law operating on a linear state feedback, similar to that of the LTI systems [2], [3]. Again, the sector bound approach is shown to be nonconservative in investigating the quantized feedback control problem. A linear matrix inequality (LMI) approach is then presented to derive the optimal quantizer under some proper coarseness definition.

\section{System Description}

As we can see from Fig. 1, a quantized feedback control system comprises three parts: a system to be controlled $(\mathbb{G})$, 
a controller $(\mathbb{K})$ and a quantizer $(\mathbb{Q})$.

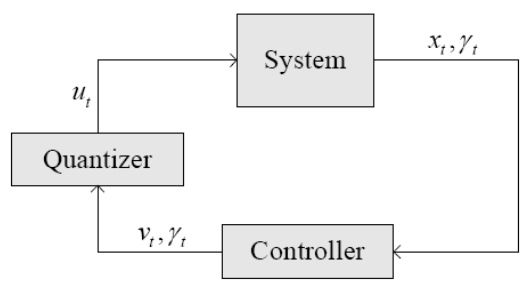

Fig. 1. Typical Quantized Feedback Control System.

In this paper, we consider a single-input MJS as follows:

$$
\mathbb{G}: \quad x_{t+1}=A_{\theta_{t}} x_{t}+B_{\theta_{t}} u_{t},
$$

where $x_{t} \in \mathcal{R}^{n}$ is the state, $u_{t} \in \mathcal{R}$ is the control input and $\theta_{t} \in \Theta \equiv\{0,1, \cdots, N\}$ is the system mode governed by a time-homogeneous Markov chain with transition probability matrix

$$
\Pi=\left(\pi_{i j}\right)_{i, j \in \Theta} \equiv\left(\operatorname{Pr}\left(\theta_{t+1}=j \mid \theta_{t}=i\right)\right)_{i, j \in \Theta} .
$$

The quantized state feedback can be denoted by

$$
\begin{array}{ll}
\mathbb{K}: & v_{t}=g\left(x_{t}, \gamma_{t}\right), \\
\mathbb{Q}: & u_{t}=f\left(v_{t}, \gamma_{t}\right),
\end{array}
$$

where $\gamma_{t} \in \Gamma$ is a mode-related input signal at the controller/quantizer side at time step $t$, which would further determine the desired form of stochastic Lyapunov function and the underlying quantization strategy. Note that $f(\cdot, \cdot)$ is assumed to be symmetric with respect to $v(t)$ for any given mode $i$, i.e., $f\left(-v_{t}, i\right)=-f\left(v_{t}, i\right)$. In the following, we deal with two cases indicated in the definition below.

Definition 2.1: The state feedback law $(\mathbb{K})$ and quantizer $(\mathbb{Q})$ defined in (3)(4) is said to be mode-dependent, if $\gamma_{t}=$ $\theta_{t}$; it is mode-independent, if there is no mode-related input signal available at $\mathbb{K} / \mathbb{Q}$ side, i.e., $v_{t}=g\left(x_{t}\right), u_{t}=f\left(v_{t}\right)$.

\section{Mode-Dependent Quantizer}

\section{A. Preliminaries}

Let $\mathcal{F}_{t}=\sigma\left\{x_{0}, \theta_{0}, \cdots, x_{t}, \theta_{t}\right\}$ for $\forall t \geq 0$, be the $\sigma$ algebra generated by $\left\{\left(x_{k}, \theta_{k}\right), 0 \leq k \leq t\right\}$.

Definition 3.1: For system

$$
x_{t+1}=h\left(x_{t}, \theta_{t}\right)
$$

with possibly nonlinear mapping $h(\cdot, \cdot)$, if for every initial condition $x_{0}, \theta_{0}, \mathrm{E}\left[\left\|x_{t}\right\|^{2} \mid x_{0}, \theta_{0}\right]$ is well-defined for any $t$, then the equilibrium point at the origin is mean square (MS) stable when $\lim _{t \rightarrow+\infty} \mathrm{E}\left[\left\|x_{t}\right\|^{2} \mid x_{0}, \theta_{0}\right]=0$.

To avoid the trivialness, we assume that system (1) with $u_{t} \equiv 0$ is not MS stable. In fact, the MS stability of the openloop system would require only the existence of a uniform quantizer with zero quantization density since zero control input could be adopted all along; also see Corollary 2.1 in [2] for LTI systems.
Definition 3.2: The equilibrium point at the origin of system (5) is mean square quadratically (MSQ) stable, if there exist a positive-definite function

$$
V_{1}\left(x_{t}, \theta_{t}\right) \equiv x_{t}^{\prime} P_{\theta_{t}} x_{t}
$$

and positive-definite matrices $Q_{\theta_{t}}$ such that

$$
\begin{aligned}
\nabla V_{1}\left(x_{t}, \theta_{t}\right) & \equiv \mathrm{E}\left[V_{1}\left(x_{t+1}, \theta_{t+1}\right)-V_{1}\left(x_{t}, \theta_{t}\right) \mid \mathcal{F}_{t}\right] \\
& =\mathrm{E}\left[V_{1}\left(x_{t+1}, \theta_{t+1}\right) \mid x_{t}, \theta_{t}\right]-V_{1}\left(x_{t}, \theta_{t}\right) \\
& <-x_{t}^{\prime} Q_{\theta_{t}} x_{t}, \quad \forall x_{t} \in \mathcal{R}^{n}, \theta_{t} \in \Theta, x_{t} \neq 0 .(7)
\end{aligned}
$$

Remark 3.1: In order to clearly reveal the connection between MSQ stability and coarsest quantization, we use $"<"$ in (7), without loss of generality, instead of " $\leq$ " as in Definition 2.2 of [13].

Following a similar proof of Theorem 1 in [14], we can get the lemma below.

Lemma 1: The MSQ stability of the equilibrium point at the origin of system (5) implies the corresponding MS stability.

The controller (3) and the quantizer (4) can be further described as

$$
\mathbb{K}: \quad v_{t}=g\left(x_{t}, \theta_{t}\right), \quad \mathbb{Q}: \quad u_{t}=f\left(v_{t}, \theta_{t}\right) .
$$

Definition $3.3(Q C L F)$ : A positive-definite quadratic function $V_{1}\left(x_{t}, \theta_{t}\right)$ of the form (6) is called a quadratic control Lyapunov function (QCLF) for system (1), if a memoryless quantized state feedback (8) exists such that the closed-loop system

$$
x_{t+1}=A_{\theta_{t}} x_{t}+B_{\theta_{t}} f\left(g\left(x_{t}, \theta_{t}\right), \theta_{t}\right)
$$

admits $V_{1}\left(x_{t}, \theta_{t}\right)$ as a parameterized Lyapunov function, i.e., the condition (7) holds for some $P_{i}, Q_{i}, \forall i \in \Theta$.

It is also worthy mentioning that system (9) is generally nonlinear, since the control signal $u_{t}$ is a nonlinear function of $x_{t}$.

Next, we introduce an assumption on $\mathbb{G}$ and generalize the definition of quantization density introduced in [2] to the MJS case.

Assumption 3.1: The unquantized system $(\mathbb{G})$ is assumed to be MS stabilizable via linear mode-dependent statefeedback law:

$$
v_{t}=K_{\theta_{t}} x_{t} .
$$

Definition 3.4: The mode density of the quantizer $f(\cdot, \cdot)$ with respect to mode $i, i \in \Theta$ is defined as $\eta_{f}(i) \equiv$ $\limsup _{\epsilon \rightarrow 0} \frac{\# l[\epsilon, i]}{-\ln \epsilon}$, where $\# l[\epsilon, i]$ is the number of quantization levels in the interval $[\epsilon, 1 / \epsilon]$ with $f(\cdot, i)$.

\section{B. Solution}

The main purpose of this paper is to solve the following problem.

Problem 3.1: For system (1) with a given QCLF $V\left(x_{t}, \theta_{t}\right)$, find $f(\cdot, \cdot)$ and $g(\cdot, \cdot)$ such that the resultant quantizer is coarsest for the given $V\left(x_{t}, \theta_{t}\right)$, i.e., there is no other set of $\bar{\eta}_{f}(i)$, such that $\bar{\eta}_{f}(i) \leq \eta_{f}(i), \forall i \in \Theta$ with at least one strict inequality and the condition (7) is satisfied for the predefined $P_{i}, Q_{i}, \forall i \in \Theta$. 
A mode-dependent quantizer is said to be logarithmic, if for any $i \in \Theta$, the corresponding set of quantized level $\mathcal{U}_{i}$ has the following form:

$$
\begin{aligned}
\mathcal{U}_{i}= & \left\{ \pm u_{l}(i): u_{l}(i)=\rho^{l}(i) u_{0}, \quad u_{0}>0,\right. \\
& \text { for } l \in \pm 1, \pm 2, \cdots\} \cup\left\{ \pm u_{0}\right\} \cup\{0\},
\end{aligned}
$$

where

$$
\rho(i)=\frac{1-\delta(i)}{1+\delta(i)}
$$

Note that there is no loss of generality by choosing the same $u_{0}$ for every $i \in \Theta$; see Lemma 2.1 in [2].

Further define

$$
\delta_{m}(i) \equiv \begin{cases}+\infty, \quad \text { for } B_{i}=0 \\ \frac{1}{\sqrt{K_{m i} M_{i}^{-1} K_{m i}^{\prime}}}, \text { for } B_{i} \neq 0\end{cases}
$$

where

$$
\begin{aligned}
K_{m i} & =-\frac{\sum_{j=0}^{N} \pi_{i j} B_{i}^{\prime} P_{j} A_{i}}{\sum_{j=0}^{N} \pi_{i j} B_{i}^{\prime} P_{j} B_{i}}, \\
M_{i}= & \frac{\sum_{j=0}^{N} \pi_{i j} A_{i}^{\prime} P_{j} B_{i} B_{i}^{\prime} P_{j} A_{i}}{\left(\sum_{j=0}^{N} \pi_{i j} B_{i}^{\prime} P_{j} B_{i}\right)^{2}} \\
& -\frac{\sum_{j=0}^{N} \pi_{i j} A_{i}^{\prime} P_{j} A_{i}-P_{i}+Q_{i}}{\sum_{j=0}^{N} \pi_{i j} B_{i}^{\prime} P_{j} B_{i}} .
\end{aligned}
$$

Then the next theorem gives a solution to Problem 3.1.

Theorem 3.1: If the system (1) with quantized state feedback (8) admits (6) as a QCLF, i.e., the condition (7) holds for some $P_{i}, Q_{i}, \forall i \in \Theta$, then the coarsest quantizer can be approached by a linear unquantized feedback law $v_{t}=K_{\theta_{t}} x_{t}$ and the following mode dependent logarithmic form:

- for the given $\theta_{t}$, if $\delta_{m}\left(\theta_{t}\right)<1$, then

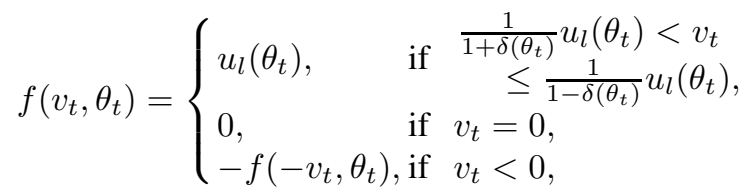

with

$$
\delta\left(\theta_{t}\right)=\delta_{m}\left(\theta_{t}\right), \quad K_{\theta_{t}}=K_{m \theta_{t}} ;
$$

- if $\delta_{m}\left(\theta_{t}\right)=1$, then

$f\left(v_{t}, \theta_{t}\right)= \begin{cases}u_{0}, & \text { if } v_{t}>\frac{1}{2} u_{0} \\ 0, & \text { if } 0 \leq v_{t} \leq \frac{1}{2} u_{0}, \\ -f\left(-v_{t}, \theta_{t}\right), & \text { if } v_{t}<0\end{cases}$

with

$$
\delta\left(\theta_{t}\right)=\delta_{m}\left(\theta_{t}\right), \quad K_{\theta_{t}}=K_{m \theta_{t}} ;
$$

- if $\delta_{m}\left(\theta_{t}\right)>1$, then

$$
f\left(v_{t}, \theta_{t}\right)=0, \quad \text { with } \delta\left(\theta_{t}\right)=1, \quad K_{\theta_{t}}=0 .
$$

Proof: Can be found in [15].

The quantization error is mode-dependent:

$$
e_{t} \equiv u_{t}-v_{t}=f\left(v_{t}, \theta_{t}\right)-v_{t}=\Delta\left(v_{t}, \theta_{t}\right) v_{t},
$$

where $\Delta\left(v_{t}, \theta_{t}\right) \in\left[-\delta\left(\theta_{t}\right), \delta\left(\theta_{t}\right)\right]$ and $\delta\left(\theta_{t}\right)$ is defined in Theorem 3.1. Then, the closed-loop quantized feedback system with $v_{t}=K_{\theta_{t}} x_{t}$ becomes the following uncertain MJS:

$$
x_{t+1}=A_{\theta_{t}} x_{t}+B_{\theta_{t}}\left(1+\Delta\left(K_{\theta_{t}} x_{t}, \theta_{t}\right)\right) K_{\theta_{t}} x_{t} .
$$

The theorem below tells us that the sector bound approach is still valid.

Theorem 3.2: Given a mode dependent logarithmic quantizer (14)-(16) with a set of fixed $0 \leq \rho(i)<1, i \in \Theta$, system (1) with quantized linear state feedback (8) has a QCLF (6) if and only if the following uncertain system:

$$
x_{t+1}=A_{\theta_{t}} x_{t}+B_{\theta_{t}}\left(1+\Delta\left(\theta_{t}\right)\right) v_{t},
$$

is robust MSQ stabilizable for uncertainty $\Delta\left(\theta_{t}\right) \in$ $\left[-\delta\left(\theta_{t}\right), \delta\left(\theta_{t}\right)\right]$ via linear state-feedback law (10), where $\rho(i)$ and $\delta(i)$ are related by (12).

Proof: Can be found in [15].

Next, we formulate the robust MSQ stability of (18) into LMIs.

Proposition 3.1: The robust MSQ stability of the system (18) with a set of $0<\delta(i) \leq 1, i \in \Theta$ and $v_{t}=K_{\theta_{t}} x_{t}$ is equivalent to

$$
\left[\begin{array}{cccc}
-S_{i} & S_{i} & Y_{i}^{\prime} & \Phi_{i} \\
* & -W_{i} & 0 & 0 \\
* & * & -\tau(i) & 0 \\
* & * & * & \Xi_{i}
\end{array}\right]<0
$$

for some variables $S_{i}>0, W_{i}>0, Y_{i}$ and $\tau(i)>0$ for $\forall i \in \Theta$, where $\Phi_{i}, \Xi_{i}$ are shown on the bottom of the next page.

Proof: Can be found in [15].

Note that (19) is convex in $S_{i}>0, W_{i}>0, Y_{i}$ and $\tau(i)>0$ for any fixed set of $\delta(i)$. It is thus possible to search desirable $0<\delta(i) \leq 1$ over constraints (19) based on some measures of data-rate, e.g., $\bar{\delta}$ in (20) defined next. However, such kind of searching may be time-consuming especially when the number of system modes $N$ is large.

\section{Examples}

In order to facilitate the quantization optimization, the measurement on sector bound could be chosen as

$$
\bar{\delta} \equiv \min _{i \in \Theta}\{\delta(i)\}
$$

which captures the worst-case quantizer among all system modes. In the following two examples, we try to characterize the quantizer with the largest sector bound $\bar{\delta}$ over all possible QCLFs.

Example 3.1: Consider a MJS (1) modified from [13]:

$$
A_{0}=A_{1}=\left[\begin{array}{cc}
0 & 1 \\
1.8 & -0.3
\end{array}\right], \quad B_{0}=\left[\begin{array}{l}
1 \\
1
\end{array}\right], \quad B_{1}=\left[\begin{array}{l}
0 \\
1
\end{array}\right] \text {. }
$$

The system matrices $A_{0}, A_{1}$ contain two unstable poles $\{1.2,-1.5\}$. Suppose the transition probability matrix is given by

$$
\Pi_{1}=\left[\begin{array}{ll}
0.1 & 0.9 \\
0.3 & 0.7
\end{array}\right]
$$


then the plant is MS stabilizable via (10), we can compute $\bar{\delta}=0.4783$ with state feedback (10) and gain matrices: $K_{0}=$ $\left[\begin{array}{ll}-0.8846 & -0.3611\end{array}\right], \quad K_{1}=\left[\begin{array}{ll}-1.8 & 1.1507\end{array}\right]$.

Next, we assume the transition probability matrix is changed to

$$
\Pi_{2}=\left[\begin{array}{ll}
0.2 & 0.8 \\
0.2 & 0.8
\end{array}\right]
$$

then we can get $\bar{\delta}=0.4698$ with gain matrices: $K_{0}=$ [-0.6728 - 0.5141], $K_{1}=\left[\begin{array}{ll}-1.8 & 1.0453\end{array}\right]$.

Example 3.2: Consider a MJS (1) with the same system matrices $A_{0}, A_{1}, B_{1}, \Pi_{2}$ given in Example 3.1 except setting $B_{0}=\left[\begin{array}{ll}0 & 0\end{array}\right]^{\prime}$. Then, we have $\bar{\delta}=0.3685$ with gain matrices: $K_{0}=\left[\begin{array}{ll}0 & 0\end{array}\right], \quad K_{1}=\left[\begin{array}{ll}-1.8 & 0.675\end{array}\right]$.

\section{Mode-IndePendent QuANTIZER}

\section{A. Preliminaries}

Assumption 4.1: In this section, the unquantized system $(\mathbb{G})$ is assumed to be MS stabilizable via linear modeindependent state-feedback law:

$$
v_{t}=K x_{t}
$$

which is also said to be strongly MS stable in literature; see e.g. [16].

The controller and the quantizer now can be expressed as

$$
\mathbb{K}: \quad v_{t}=g\left(x_{t}\right), \mathbb{Q}: \quad u_{t}=f\left(v_{t}\right) .
$$

In contrast to (6), for the mode-independent scheme the Lyapunov function is chosen as

$$
V_{2}\left(x_{t}\right) \equiv x_{t}^{\prime} P x_{t}
$$

Definition 4.1: The equilibrium point at the origin of system (5) is strongly MSQ stable, if there exist a positivedefinite function $V_{2}\left(x_{t}\right)$ in (26) and positive-definite matrix $Q$ such that

$$
\begin{aligned}
\nabla V_{2}\left(x_{t}\right) & =\mathrm{E}\left[V_{1}\left(x_{t+1}\right) \mid x_{t}\right]-V_{1}\left(x_{t}\right) \\
& <-x_{t}^{\prime} Q x_{t}, \quad \forall x_{t} \in \mathcal{R}^{n}, \theta_{t} \in \Theta, x_{t} \neq 0 .
\end{aligned}
$$

Assumption 4.2: The transition probability matrix (2) satisfies $\pi_{i j}=\pi_{j}$ for $\forall i, j \in \Theta$.

The above assumption implies that the system mode process is governed by an i.i.d. process (a special case of Markov chain), which would facilitate our further deduction. For autonomous jump linear system driven by a finite-state homogenous Markov chain, the Lypunov functions $V_{1}\left(x_{t}, \theta_{t}\right)$ is equivalent to $V_{2}\left(x_{t}\right)$ when the Markov chain degenerates to the i.i.d. process [17].

\section{B. Solution}

Based on (26) we can easily get

$$
\begin{aligned}
\nabla V_{2}(x)= & \sum_{j=0}^{N} \pi_{j} x^{\prime}\left(A_{j}^{\prime} P A_{j}-P\right) x+2 x^{\prime} \sum_{j=0}^{N} \pi_{j} A_{j}^{\prime} P B_{j} u \\
& +u^{\prime} \sum_{j=0}^{N} \pi_{j} B_{j}^{\prime} P B_{j} u
\end{aligned}
$$

A mode-independent quantizer is called logarithmic if the set of quantized level $\mathcal{U}$ has the following form:

$\mathcal{U}=\left\{ \pm u_{l}: u_{l}=\rho^{l} u_{0}, \quad u_{0}>0\right.$, for $\left.l \in \pm 1, \pm 2, \cdots\right\}$

$$
\cup\left\{ \pm u_{0}\right\} \cup\{0\} \text {. }
$$

The corresponding quantizer is given by

$$
f\left(v_{t}\right)= \begin{cases}u_{l}, & \text { if } \frac{1}{1+\delta} u_{l}<v_{t} \leq \frac{1}{1-\delta} u_{l} \\ 0, & \text { if } v_{k}=0 \\ -f\left(-v_{t}\right), & \text { if } v_{t}<0\end{cases}
$$

where

$$
\rho=\frac{1-\delta}{1+\delta}
$$

Define $\delta_{m} \equiv \frac{1}{\sqrt{K_{m} M^{-1} K_{m}^{\prime}}}$, where

$$
K_{m} \equiv-\frac{\sum_{j=0}^{N} \pi_{j} B_{j}^{\prime} P A_{j}}{\sum_{j=0}^{N} \pi_{j} B_{j}^{\prime} P B_{j}}
$$

$M \equiv \frac{\sum_{j=0}^{N} \pi_{j} A_{j}^{\prime} P B_{j} B_{j}^{\prime} P A_{j}}{\left(\sum_{j=0}^{N} \pi_{j} B_{j}^{\prime} P B_{j}\right)^{2}}-\frac{\sum_{j=0}^{N} \pi_{j} A_{j}^{\prime} P A_{j}-P+Q}{\sum_{j=0}^{N} \pi_{j} B_{j}^{\prime} P B_{j}}$.

The following theorem can be proved similarly to Theorem 3.1 and 3.2.

Theorem 4.1: (a). If the system (1) under Assumption 4.2 with quantized state feedback (25) admits (26) as a QCLF, i.e., the condition (27) holds for some $P, Q$, then the coarsest quantizer can be approached by a linear unquantized feedback law $v_{t}=K x_{t}$ and the mode-independent logarithmic form (30) with $K=K_{m}$ and $\delta=\delta_{m}$.

(b). Given a mode-independent logarithmic quantizer (30) with a fixed $\rho \in[0,1)$, system (1) with quantized linear state feedback (25) has a QCLF (26) if and only if the following uncertain system:

$$
x_{t+1}=A_{\theta_{t}} x_{t}+B_{\theta_{t}}(1+\Delta) v_{t},
$$

is robust strongly MSQ stabilizable for uncertainty $\Delta \in$ $[-\delta, \delta]$ via linear state-feedback law (24), where $\rho$ and $\delta$ are related by (31).

$$
\begin{gathered}
\Phi_{i}=\left[\sqrt{\pi_{i 0}}\left(S_{i} A_{i}^{\prime}+Y_{i}^{\prime} B_{i}^{\prime}\right)\right. \\
\Xi_{i}=\left[\begin{array}{cccc}
-S_{0}+\tau(i) \delta(i)^{2} \pi_{i 0} B_{i} B_{i}^{\prime} & \tau(i) \delta(i)^{2} \sqrt{\pi_{i 0} \pi_{i 1}} B_{i} B_{i}^{\prime} & \cdots & \tau(i) \delta(i)^{2} \sqrt{\pi_{i 0} \pi_{i N}} B_{i} B_{i}^{\prime} \\
* & -S_{1}+\tau(i) \delta(i)^{2} \pi_{i 1} B_{i} B_{i}^{\prime} & \cdots & \tau(i) \delta(i)^{2} \sqrt{\pi_{i 1} \pi_{i N}} B_{i} B_{i}^{\prime} \\
\vdots & \vdots & \ddots & \vdots \\
* & * & \cdots & -S_{N}+\tau(i) \delta(i)^{2} \pi_{i N} B_{i} B_{i}^{\prime}
\end{array}\right] .
\end{gathered}
$$


Obviously, the density for quantizer (30) is $\eta_{f}=-\frac{2}{\ln (\rho)}$ with $\rho$ defined in Theorem 4.1.

Theorem 4.2: The least density $\underline{\eta}_{f}=-\frac{2}{\ln (\underline{\rho})}$ can be obtained through the optimization $\bar{\delta}^{2} \equiv \max _{S, W, Y}\left\{\delta^{2}\right\}$ over the constraint

$$
\left[\begin{array}{cccc}
-S & S & Y^{\prime} & \Phi \\
* & -W & 0 & 0 \\
* & * & -1 & 0 \\
* & * & * & \Xi
\end{array}\right]<0,
$$

with variables $S>0, W>0, Y$ and $\delta^{2}$, where $\Phi, \Xi$ are shown on the bottom of the next page. Note that $\underline{\rho}$ and $\bar{\delta}$ are related by (31) and $K=Y S^{-1}$.

Proof: It is easy to get the result by taking $S=P^{-1} / \tau, W=$ $Q^{-1} / \tau$ and $Y=K S$, where $\tau$ is the scaling variable.

In contrast to (19) in the form of $N+1$ coupled constrains, Theorem 4.2 only contains one single constraint without linear searching, and thus the computational burden would be eased.

Example 4.1: Suppose $A_{0}, A_{1}, B_{0}, B_{1}, \Pi_{2}$ are given in Example 3.1. By Theorem 4.2, $\bar{\delta}=0.3873$ with state feedback (24) and gain matrix $K=\left[\begin{array}{ll}-1.682 & 0.8854\end{array}\right]$.

Remark 4.1: By comparing Example 3.1 with 4.1, we can see that mode-dependent pattern presents a less conservative but more computationally demanding result with larger $\bar{\delta}$. It is understandable, as the quantizer (14)-(16) takes into account the system mode information.

\section{Application to NCS with UDP-Like Channel}

UDP-like here means that there is no acknowledgment signal with respect to data transmission through unreliable networks, which falls into the mode-independent pattern. Two NCS structures are considered as follows.

1) Discrete Plant with Zero-Control Strategy and Binary Dropouts: A quantized feedback NCS with UDP-like channel is shown in Fig. 2, where the network $(\mathbb{N})$ is an analog multiplicative memoryless channel associated to $\theta$ :

$$
\mathbb{N}: \quad w_{t}=\theta_{t} u_{t},
$$

and $\theta_{t} \in \Theta=\{0,1\}$ is an i.i.d. random variable: $\operatorname{Pr}\left(\theta_{t}=\right.$ $0)=\alpha, \quad \operatorname{Pr}\left(\theta_{t}=1\right)=1-\alpha$. As a result, the system adopted an unreliable network with packet-dropout rate $\alpha$ and a zero-control strategy.

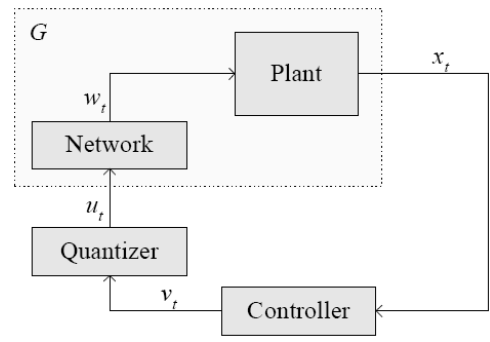

Fig. 2. Quantized Networked Control System with Binary Packet Losses.

The LTI plant $(\mathbb{P})$ is described as

$$
\mathbb{P}: \quad x_{t+1}=A x_{t}+B w_{t},
$$

and the jump system $(\mathbb{G})$ is a combination of network and LTI plant with the following system matrices:

$$
A_{0}=A_{1}=A, \quad B_{0}=0, B_{1}=B .
$$

From Theorem 4.1, we have

$$
\begin{gathered}
K_{m}=-\frac{B^{\prime} P A}{B^{\prime} P B}, \\
M=\frac{(1-\alpha) A^{\prime} P B B^{\prime} P A}{\left((1-\alpha) B^{\prime} P B\right)^{2}}-\frac{A^{\prime} P A-P+Q}{(1-\alpha) B^{\prime} P B} .
\end{gathered}
$$

Under this situation, the inequality (33) or the condition $\nabla V_{2}(x)<-x^{\prime} Q x, \forall x \neq 0$ for system (32) with control law (24) can be rewritten in the following modified Riccati inequality:

$A^{\prime} P A-P+Q-(1-\alpha)\left(1-\delta^{2}\right) A^{\prime} P B\left(B^{\prime} P B\right)^{-1} B^{\prime} P A<0$.

Based on Lemma 5.4 [12] for modified algebraic Riccati equation, $\alpha$ and $\delta$ should satisfy the following condition in order to ensure the existence of $P>0$ to (39):

$$
(1-\alpha)\left(1-\delta^{2}\right)>1-\frac{1}{\Pi_{i}\left|\lambda_{i}^{u}(A)\right|^{2}},
$$

where $\lambda_{i}^{u}(A)$ denote the unstable poles of $A$. It is easy to check the above result is consistent with Theorem 2.1 of [13], which can be seen as a special case of Theorem 4.2 in this paper.

Example 4.2: Consider a LTI system (35) with

$$
A=\left[\begin{array}{cc}
0 & 1 \\
1.8 & -0.3
\end{array}\right], \quad B=\left[\begin{array}{l}
0 \\
1
\end{array}\right] \text {. }
$$

Then the system matrices $A_{0}, A_{1}, B_{0}, B_{1}$ are the same as in Example 3.2. Assume $\alpha=0.2$, which is consistent with $\Pi_{2}$ in (23). The plant is strongly MS stabilizable, and based on Theorem 4.2 or (40), it follows that $\bar{\delta}=0.3685$ with $K=\left[\begin{array}{ll}-1.8 & 0.675\end{array}\right]$.

Remark 4.2: For system (35) with i.i.d. packet losses and zero-control strategy, it is redundant to know any system mode information. Furthermore, although Theorem 3.1 exhibits theoretical importance in quantization of MJSs, it is unsuitable for solving the quantization problem over unreliable channel, since it is unrealistic for the quantizer to know whether the current packet will be lost or not before the packet is sent over the network, which is also true for the following bounded packet loss case.

2) Continuous Plant with $\mathrm{ZOH}$ and Bounded Dropouts: In Fig. 3, the sampler is clock-driven, while the zero-order hold $(\mathrm{ZOH})$ is event-driven. The continuous-time plant together with the $\mathrm{ZOH}$ and the sampler can be expressed in the general discrete-time form (35).

Let $\left\{i_{k}, k \geq 0\right\}$ be a strictly monotonically increasing subset of $\{0,1, \cdots\}$, representing the sequence of time instants at which the data packets are successfully transmitted through the network $(\mathbb{N})$. Without loss of generality, set $i_{0}=0$. The packet-loss process is defined as

$$
\eta\left(i_{k}\right) \equiv i_{k+1}-i_{k}-1, \quad k \geq 0 .
$$




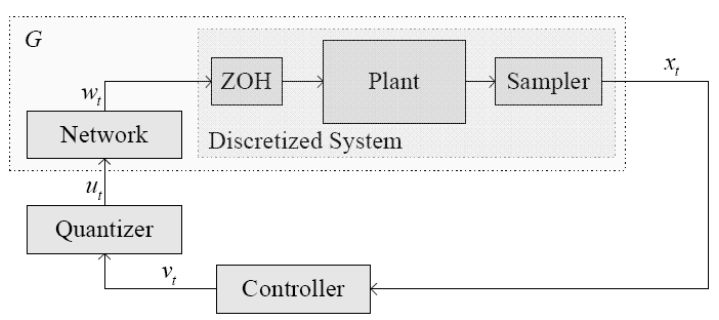

Fig. 3. Quantized Networked Control System with Bounded Packet Losses.

Here, $\eta\left(i_{k}\right)$ is driven by an i.i.d. process with finite-state range set $\Theta=\{0,1, \cdots, N\}$, where $\eta\left(i_{k}\right)=0$ indicates that there is no packet dropout during the time interval $\left(i_{k}, i_{k+1}\right]$ and $N$ represents the maximal length of consecutive packet losses. The transition probability matrix is the same as in Assumption 4.2. Then, from Theorem 9 of [7] the system $\mathbb{G}$ in Fig. 3 can be modeled as a MJS (1) with

$$
A_{i}=A^{i+1}, \quad B_{i}=\sum_{r=0}^{i} A^{r} B .
$$

Thus, Theorems 4.1 and 4.2 can be applied as shown in the numerical example borrowed from [7].

Example 4.3: The discretized system in Fig. 3 is (35) with

$$
A=\left[\begin{array}{ccc}
0.6065 & 0 & -0.2258 \\
0.3445 & 0.7788 & -0.0536 \\
0 & 0 & 1.2840
\end{array}\right], \quad B=\left[\begin{array}{c}
-0.0582 \\
-0.0093 \\
0.5681
\end{array}\right] \text {. }
$$

Suppose the maximum consecutive packet dropouts of the underlying network is $N=4$ and the transition probability matrix (2) under Assumption 4.2 is given by:

$$
\pi_{0}=0.5, \quad \pi_{1}=0.2, \quad \pi_{2}=0.1, \quad \pi_{3}=0.1, \quad \pi_{4}=0.1 .
$$

By using Theorem 4.2 with system matrices (43), it follows that $\bar{\delta}=0.4085$ with $K=\left[\begin{array}{lll}0 & 0 & -0.8452\end{array}\right]$.

\section{CONClusions}

This paper has shown that for linear systems with Markovian jump parameters, mode-dependent (or modeindependent) logarithmic quantizer is still optimal in MSQ (or strongly MSQ) stability sense, and the sector bound approach again provides a nonconservative way for studying the corresponding design problems. It is also worthy noting that the mode-dependent or mode-independent QCLF in this paper can be modified into other complicated forms, e.g. quantization-error-dependent form or polytopic form, in order to achieve less conservative results (smaller $\underline{\eta}_{f}$ or larger $\bar{\delta})$ at the expenses of additional computational complexity. Possible future work includes output feedback stabilization problem, $H_{\infty}$ and quadratic performance analysis, and generalization to the MIMO system case.

\section{REFERENCES}

[1] R. Kalman, "Nonlinear aspects of sampled-data control systems," Proceedings, Symposium on Nonlinear Circuit Analysis VI, pp. 273313, 1956.

[2] N. Elia and S. Mitter, "Stabilization of linear systems with limited information," Automatic Control, IEEE Transactions on, vol. 46, no. 9, pp. 1384-1400, 2001

[3] M. Fu and L. Xie, "The sector bound approach to quantized feedback control," Automatic Control, IEEE Transactions on, vol. 50, no. 11 pp. 1698-1711, 2005.

[4] H. Gao and T. Chen, "A new approach to quantized feedback control systems," Automatica, vol. 44, no. 2, pp. 534-542, 2008.

[5] S. Hu and W. Yan, "Stability robustness of networked control systems with respect to packet loss," Automatica, vol. 43, no. 7, pp. 1243-1248, 2007.

[6] P. Seiler and R. Sengupta, "An $H_{\infty}$ approach to networked control," Automatic Control, IEEE Transactions on, vol. 50, no. 3, pp. 356-364, 2005.

[7] J. Xiong and J. Lam, "Stabilization of linear systems over networks with bounded packet loss," Automatica, vol. 43, no. 1, pp. 80-87, 2007.

[8] N. Elia, "Remote stabilization over fading channels," Systems \& Control Letters, vol. 54, no. 3, pp. 237-249, 2005.

[9] B. Sinopoli, L. Schenato, M. Franceschetti, K. Poolla, M. Jordan, and S. Sastry, "Kalman filtering with intermittent observations," Automatic Control, IEEE Transactions on, vol. 49, no. 9, pp. 1453-1464, 2004.

[10] M. Huang and S. Dey, "Stability of Kalman filtering with Markovian packet losses," Automatica, vol. 43, no. 4, pp. 598-607, 2007.

[11] L. Xie and L. Xie, "Peak covariance stability of a random Riccati equation arising from Kalman filtering with observation losses," Journal of Systems Science and Complexity, vol. 20, no. 2, pp. 262-272, 2007.

[12] L. Schenato, B. Sinopoli, M. Franceschetti, K. Poolla, and S. Sastry, "Foundations of control and estimation over lossy networks," Proceedings of the IEEE, vol. 95, no. 1, pp. 163-187, 2007.

[13] H. Hoshina, K. Tsumura, and H. Ishii, "The coarsest logarithmic quantizers for stabilization of linear systems with packet losses," Decision and Control, 46th IEEE Conference on, pp. 2235-2240, 2007.

[14] E. Boukas and Z. Liu, "Robust $H_{\infty}$ control of discrete-time Markovian jump linear systems with mode-dependent time-delays," Automatic Control, IEEE Transactions on, vol. 46, no. 12, pp. 1918-1924, 2001.

[15] N. Xiao, L. Xie, and M. Fu, "Quantized stabilization of Markov jump linear systems," Nanyang Technological University, Singapore, Tech. Rep., 2009. [Online]. Available: http://www3.ntu.edu.sg/home2007/ xiao0023/TRQSMJL09.pdf

[16] C. de Souza, "Robust stability and stabilization of uncertain discretetime Markovian jump linear systems," Automatic Control, IEEE Transactions on, vol. 51, no. 5, pp. 836-841, 2006.

[17] Y. Fang and K. Loparo, "Stochastic stability of jump linear systems," Automatic Control, IEEE Transactions on, vol. 47, no. 7, pp. 1204$1208,2002$.

$$
\begin{aligned}
& \Phi=\left[\sqrt{\pi_{0}}\left(S A_{0}^{\prime}+Y^{\prime} B_{0}^{\prime}\right) \quad \sqrt{\pi_{1}}\left(S A_{1}^{\prime}+Y^{\prime} B_{1}^{\prime}\right) \quad \cdots \quad \sqrt{\pi_{N}}\left(S A_{N}^{\prime}+Y^{\prime} B_{N}^{\prime}\right)\right], \\
& \Xi=\left[\begin{array}{cccc}
-S+\delta^{2} \pi_{0} B_{0} B_{0}^{\prime} & \delta^{2} \sqrt{\pi_{0} \pi_{1}} B_{0} B_{1}^{\prime} & \cdots & \delta^{2} \sqrt{\pi_{0} \pi_{N}} B_{0} B_{N}^{\prime} \\
* & -S+\delta^{2} \pi_{1} B_{1} B_{1}^{\prime} & \cdots & \delta^{2} \sqrt{\pi_{1} \pi_{N}} B_{1} B_{N}^{\prime} \\
\vdots & \vdots & \ddots & \vdots \\
* & * & \cdots & -S+\delta^{2} \pi_{N} B_{N} B_{N}^{\prime}
\end{array}\right] .
\end{aligned}
$$

